\title{
Bispecifics and antibody-drug conjugates: a positive synergy
}

\author{
Antoine Maruani* \\ Department of Chemistry, University College London, London, UK
}

\begin{abstract}
Bispecific antibodies (BsAbs) are antibodies with two different paratopes. In the past decade, advances in protein engineering have enabled the development of more than 100 formats of BsAbs. With two BsAbs approved for therapeutic use and more than 60 in clinical trials, this research area has shifted from being effervescent to being a mainstream therapeutic development topic. In parallel, recent progress in protein conjugation and cytotoxicity of small molecule drugs has resulted in a boom in monospecific antibody therapeutics development such as antibody-drug conjugates (ADCs). Recent examples have demonstrated how BsAbs approaches can be used to generate ADCs with better efficacy and safety profile. Rather than examining these two different yet similar areas independently, this minireview will explore the potential synergies that can exist between them.
\end{abstract}

Keywords: Bispecifics, Biparatopic, Antibody-Drug Conjugate (ADC), Therapeutic Index

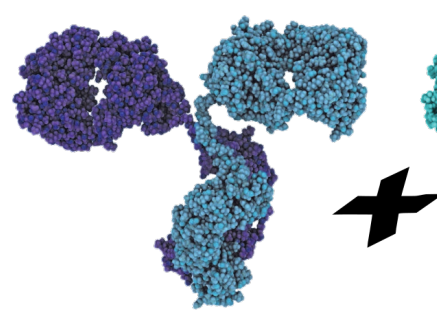

Bispecific

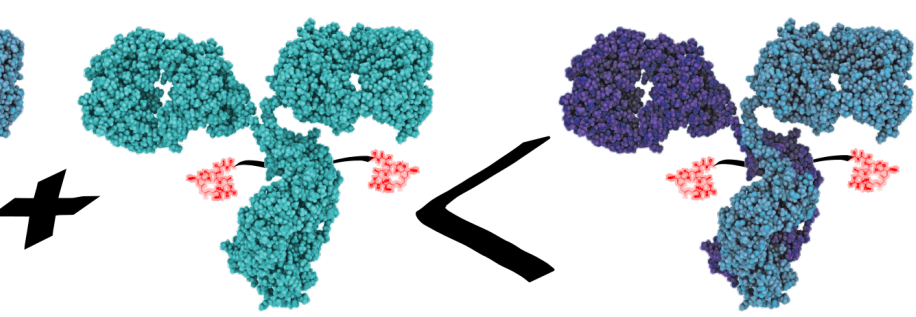

ADC
Bispecific ADC

\footnotetext{
* Corresponding author

Email address: antoine.maruani@ucl.ac.uk (Antoine Maruani)
} 


\section{Contents}

1 Introduction $\quad 3$

1.1 Antibody-drug conjugates . . . . . . . . . . . . . . . . 3

1.2 Combination therapies . . . . . . . . . . . . . . . 3

1.3 Bispecific antibodies . . . . . . . . . . . . . . . . 4

1.3.1 Production - early days . . . . . . . . . . . . . . . . . 4

1.3.2 State of the market . . . . . . . . . . . . . . 5

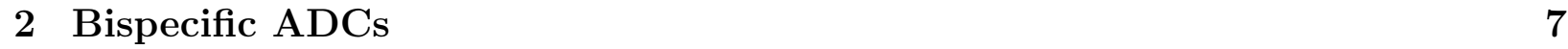

2.1 Selectivity . . . . . . . . . . . . . . . . . . 7

2.2 Endocytosis and lysosomal trafficking . . . . . . . . . . . . . . . 8

2.2.1 Fast-internalising receptor . . . . . . . . . . . . . . . . 9

2.2.2 Crosslinking and clustering of receptors . . . . . . . . . . . . . . 11

3 Conclusion $\quad 12$ 


\section{Introduction}

Despite the continual development of new therapeutic strategies based on monoclonal antibodies (mAbs), in oncology in particular, certain limitations to their therapeutic and diagnostic use remain. For 40 years, advances in the modification of their format (e.g. use of fragments), their production method and their functionalisation (e.g. antibody-drug conjugates) have led to improvements in their efficacy and have overcome many existing barriers. Nevertheless, despite these advances, the safety and efficacy profile of this class of molecules is still suboptimal; broadening their therapeutic index remains necessary. Hence, it is relevant to ask what could become of these mAbs in the future and how they could be improved further to effectively target cancer cells whilst causing minimal off-site toxicity to normal tissue.

\subsection{Antibody-drug conjugates}

Amongst the most advanced applications of mAbs, antibody-drug conjugates (ADCs) are designed to selectively deliver potent cytotoxic drugs within cancer cells expressing the target surface antigen. ADCs are composed of one or more cytotoxic agents connected to a mAb by a covalent bond. The first conjugated antibodies were composed of murine mAbs covalently bound to cytotoxic agents (e.g. doxorubicin, vinblastine and methotrexate) [1]. These conjugated antibodies were evaluated in early clinical trials, with little success because of insufficient immunogenicity, lack of potency, and selectivity against tumour antigens. The lessons learned from these explorations have enabled optimisation of the design and performance of these compounds and have led to a renewed interest in this technology. This led to the approval by the FDA of 4 ADCs and to more than 70 currently in clinical trials.

\subsection{Combination therapies}

Most of the mAbs and ADCs in clinical trials and on the market can interact with only one target on the cancer cell. However, most complex diseases are multifactorial, with redundancies and synergies between different pathways; this monospecificity often reveals unsuited. The targeting of multiple entities could therefore improve the efficacy of treatment 
and counteract mechanisms of tumour survival. Strategies combining multiple mAbs directed against different targets are being considered. For example, the concomitant injection of trastuzumab (anti-HER2) and bevacizumab (anti-VEGF-A) has shown encouraging results in HER2 + metastatic breast cancer [2]. However, other combinations may lead to unfavourable results, e.g. bevacizumab with cetuximab or panitumumab in advanced colorectal cancer [3]. The simultaneous use of two antibodies directed against the same target but on different epitopes is another potential strategy. Sym004 (a 1:1 mixture of two recombinant humanmouse chimeric mAbs directed against non-overlapping epitopes of the EGFR) showed encouraging results in patients with squamous cell cancer of the head and neck who have become resistant to initial therapy [4]. The different published studies on combination therapies with mAbs have shown widely varying results [3]. More preclinical and clinical studies are necessary to decipher and understand the mechanisms behind synergistic or antagonistic effects of combining mAbs.

\subsection{Bispecific antibodies}

Following this combinatorial concept, a new class of therapeutics emerged: bispecific antibodies (BsAbs). The principle behind BsAbs is the targeting of two molecules through a single platform to take into account the multifactorial side of diseases and simultaneously affect multiple pathways to increase efficacy [5].

\subsubsection{Production - early days}

Bispecific antibodies are not natively found in organisms, they must be produced artificially. The first concepts for BsAbs date from the mid-1980s [6, 7]. As a result, early production was highly complicated (e.g. Quadroma technique, disulfide scrambling), which led to random matching of heavy and light chains that resulted in the formation of a high proportion of poorly assembled and therefore inactive antibodies. This complexity and inefficiencies in the preparation of BsAbs greatly restricted the further development of this concept for many years.

Clinical results with the first generation of BsAbs failed to meet initial expectations [8]. This was due to their low efficacy - partly resulting from a very short plasma half-life of 
the hybrid antibodies [9] - and their immunogenicity. The Quadroma antibodies showed a therapeutic effect in some of the patients in initial clinical trials [10-12]. However, due to the murine components of the bispecific antibody, immunogenicity resulted in a human anti-mouse antibody response, with the release of human-anti-bispecific antibodies. Along with other Fc-related side effects [13-15], this precluded a longer treatment with this type of bispecific therapies [16].

Better results, with fewer side effects than with Quadroma antibodies, could be achieved with chemically linked bispecific Fab fragments. The clinical trials were moderately promising $[8,17-20]$, but ultimately the costly manufacturing process ultimately prevented further clinical studies (phase III). Interest in the concept of BsAbs eased markedly in the following years. However, thanks to advances in molecular engineering (e.g. recombinant DNA technologies), the production of BsAbs in sufficient quantity and quality is now possible and is compatible with commercialisation [21].

\subsubsection{State of the market}

To date, only three bispecifics have been approved for human treatment. In 2009, catumaxomab, an anti-CD3/anti-EpCAM BsAb used in the treatment of malignant ascites (a condition occurring in people with metastasising cancer) was the first-in-class approved by the European Union. Although it was withdrawn from the US and EU market a few years later, it demonstrated the feasibility of marketing such therapeutics, thus paving the way for further bispecifics development.

Blinatumomab, which is directed against CD3 and CD19, was approved in 2014 by the US Food and Drug Administration (FDA) and by the EU the following year. It is used in patients with late stages of non-Hodgkin's lymphoma [22] and in patients with relapsed or refractory B-cell precursor acute lymphoblastic leukemia.

In November 2017, emicizumab was approved under the breakthrough therapy designation by the FDA for treatment of haemophilia A. It was initially approved for patients who had developed resistance to other treatments but was then extended in April 2018 for patients 
without resistance. The data submitted for EU approval was also examined under the accelerated procedure and the EU approval took place in February 2018.

When looking at both ADC and bispecifics development simultaneously, many parallels can be drawn. For example, the 4 FDA-approved ADCs and the 3 bispecifics that have demonstrated success in the clinic to date reflect a similar presence in the current market; the wide variety of bispecific formats [21] highlights the same research efforts as the diversity of site-specific linker technologies for ADCs [23]; the fine balancing act between target, linker stability and payload cytotoxicity in ADC development is matched by the choice of format and the fine tuning of the interactions between 2 or more targets in bispecific therapeutics (Table 1).

\begin{tabular}{lll}
\hline & BsAbs & ADCs \\
\cline { 2 - 3 } First test on human & 1990 's & 1980 's \\
Clinical trials (Phases I-III) & $c a .60$ & $c a .70$ \\
Approved to date & 3 & 4 \\
Number of formats/linkers & $>100$ & $>100$ \\
\hline
\end{tabular}

Table 1: Comparison of data for BsAbs and ADCs development [17, 21, 23, 24].

Rather than advancing independently, the ADC and BsAb strategies could benefit from one another to address the remaining issues in targeted delivery of payloads (e.g. safety, efficacy, production). The past decade has seen many key improvements in the design of cytotoxic payloads and in linker technologies. These were essential to increase homogeneity and potency of ADCs and have been extensively reviewed in the last few years (see [23], [24] and [25] for example). This short review will describe recent improvements on the remaining two key components to consider when designing an ADC, the target and the antibody. It will highlight some of the bispecific strategies currently being developed to refine ADC constructs and improve their therapeutic index. 


\section{Bispecific ADCs}

Two topical points in oncology for developing bispecifics are:

- the direct targeting of two tumour antigens or two different epitopes on the same antigen (i.e. biparatopic antibody) playing an important role at the origin of the pathology (e.g. soluble or surface receptors)

- the use of antibody as a vector allowing the redirection of molecular effectors (e.g. cytotoxic drugs, radionuclides) or cellular effectors of the immune system that lack Fc receptors and are not recruited by mAbs (e.g. T cells, NK cells) to the target cells.

Not all these strategies can be adapted to ADC development and implemented to improve their pharmacological properties. Nevertheless, it has been recently demonstrated that ADC selectivity, internalisation, payload delivery, and acquired resistance can benefit greatly from bispecific approaches [24].

\subsection{Selectivity}

The ideal target antigen for an ADC would be highly expressed solely on diseased tissue. Unfortunately, this is rarely the case and basal expression can lead to poor tumour selectivity compared to normal tissue which often results in undesired on-target toxicity [26]. In this situation, bispecific approaches can be used to allow ADCs to target cells with low antigen expression and to discriminate more efficiently between healthy and diseased tissues.

Due to expression in the normal skin cells, EGFR is a common target that can suffer from on-target toxicity, and ADCs targeting EGFR generally require additional safety assessments to limit undesired effects. Sellmann et al. demonstrated high in vitro selectivity towards tumour cells overexpressing both EGFR and c-Met by introducing a reduction in EGFR affinity in a EGFR $\times$ c-Met bispecific [27]. After sortase-mediated site-directed conjugation with tubulin inhibitor monomethyl auristatin E (MMAE), the bispecific ADC obtained was shown to have potent anti-tumour efficacy and reduced cytotoxicity toward human keratinocytes. The authors suggested that this strategy could potentially broaden the 
therapeutic window for an ADC and more generally increase the space of available targets for this class of therapeutic.

This concept of affinity-optimised binders was further developed (without cytotoxic payload) by Mazor et al. [28]. The authors reached similar conclusions by comparing a series of monovalent and bivalent BsAbs composed of trastuzumab (anti-HER2 antibody) arm paired with affinity-modulated regions of GA201 (anti-EGFR antibody). They demonstrated the pivotal roles played by the affinity of the individual arms, overall avidity and format valence. By carefully evaluating monovalent and bivalent bispecifics with low to high affinity GA201 variant, they showed that monovalent bispecifics with low affinity could selectively target and eradicate double-positive target tumours. Importantly, optimised BsAbs had little effect on single-positive, isogenic non-target tumours and high affinity or bivalent variants had none. They are currently applying this approach with bispecific ADCs to determine improvements in safety and efficacy.

\subsection{Endocytosis and lysosomal trafficking}

Besides high tumour selectivity, ADCs require efficient internalisation and trafficking to lysosomes to be efficacious. Even with a target highly expressed on a cell surface, if the ADC cannot enter the cell to deliver the payload, the treatment will have limited effect.

Due to overexpression in breast and gastric cancers compared to normal issues, HER2 is a good target candidate for ADCs. Furthermore, as HER2 is internalised, it provides an entry point for lysosomal delivery of cargos. However, HER2 is also very efficiently recycled back to the plasma membrane [29-31], thus when HER2-targeted ADCs bind to their target, they are internalised but mostly do not reach the lysosome [30]; they are recycled, which greatly limits drug release and overall efficacy of the treatment. This rapid endocytic recycling and limited lysosomal trafficking is a key issue and has been put forward as one of the potential reasons for the disappointing clinical results in several trials of ado-trastuzumab emtansine (T-DM1), an anti-HER2 ADC. To alleviate this limitation, bispecific-based strategies have been developed. Over the past decade, two main approaches have emerged to promote endocytosis and lysosomal trafficking. 


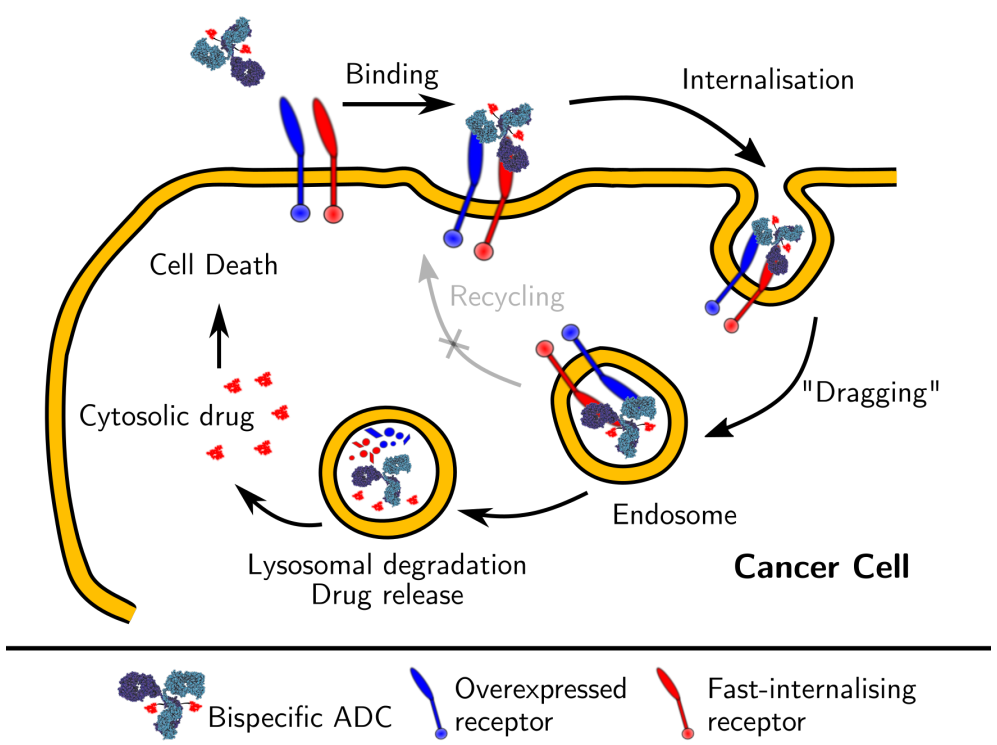

Figure 1: Schematic mechanism of action of bispecific ADCs relying on fast internalising receptors to migrate to the lysosome and deliver their payload.

\subsubsection{Fast-internalising receptor}

The first approach consists in utilising one arm of the bispecific to target an existing fast internalising receptor that promotes trafficking to lysosome and degradation of the complex whilst the second arm is used to enable specific binding to the surface of the target tumour cell. This approach alleviates the need to focus solely on internalising and non fast-recycling targets for cancer therapy but still requires the presence of a strongly internalising target on the cell surface to "drag" the other antigen and transfer it to the lysosome (Figure 1) [32].

In 2016, Lee et al. generated novel tetravalent c-Met $\times$ EGFR or c-Met $\times$ HER2 BsAbs by combining an anti-Met antibody that induces a rapid internalisation and degradation of Met with an anti-EGFR or HER2 single-chain variable fragment [32]. Although no cytotoxic drug was attached to the BsAbs, the authors demonstrated the "dragging" capacity of their constructs thus providing a proof-of-concept for this approach. Initially developed to address resistance issues in certain cancers, this method was later applied to the preparation of ADCs with improved tumour internalisation. 
Andreev et al. compared the trafficking of HER2 with that of prolactin receptor (PRLR), another potential target in breast cancer and found that PRLR rapidly internalises and efficiently reaches lysosomes where it is degraded [33]. They reported that non-covalently crosslinking HER2 to PRLR at the cell surface, using a BsAb that binds to both receptors, dramatically enhances the degradation of HER2. Following these observations, the authors prepared a HER $2 \times$ PRLR bispecific ADC and compared it with HER2 ADC, PRLR ADC, or HER2 ADC + PRLR ADC in cell killing assays. The armed bispecific was shown to induce lysosomal trafficking of HER2 and therefore was more efficient at inducing cell-cycle arrest in breast cancer cells that coexpress intermediate HER2 and low PRLR levels. This suggests that this approach could provide benefit in this subpopulation of breast cancer patients.

Similarly, CD63, a well-established component of the late endosomal and lysosomal membranes that travels between the plasma membrane and the intracellular compartments, is an attractive target for such a strategy. This high-turnover surface protein was used in a HER $2 \times$ CD63 bispecific ADC prepared by de Goeij et al. [34]. The binding affinity of the CD63 arm was limited in order to reduce monovalent target binding and prevent uptake by healthy tissues [35], thus relatively increasing the targeting properties of the bsAb to tumour cells that coexpress HER2 and CD63. The bispecific was armed using lysine conjugation with antimitotic agent duostatin-3 attached to a lysosomal protease-cleavable linker. It demonstrated potent cytotoxicity against HER2-positive tumours in vitro and in vivo, which was not observed with monovalent HER2- and CD63-specific ADCs.

Interestingly, whilst demonstrating the potential of such a "drag and degradation" approach, DeVay et al. noted limitations to this strategy [36]. A model bispecific ADC with a HER2 arm and an Amyloid Precursor Like Protein 2 (APLP2) arm was prepared. APLP2 is overexpressed in a variety of cancer cell lines; it has been shown to travel directly to lysosomes following endocytosis and is therefore a potential suitable target [37]. The effect of APLP $2 \times$ HER 2 ADC was tested on cell lines expressing various numbers of HER2 receptors; although it generally outperformed the monospecific controls in cell lines where ratios of 
APLP2:HER2 were too extreme in either direction, it did not show a change in efficacy in vitro or in vivo relative to bivalent HER2 ADC control. The investigators speculated that the gain in internalisation due to APLP2 was offset by the loss of HER2 dimerisation which is also known to enhance internalisation and trafficking. They concluded that the approach might be useful for other targets where bivalency does not affect internalisation and that it would make a more significant impact in lower expressing cell lines.

Whilst improving endocytosis and lysosomal trafficking by targeting a protein that can act as a "carrier" for the antigen of interest is a suitable approach to increase potency and therapeutic window in $\mathrm{ADC}$, it requires careful selection of both targets and cell population to be suitable.

\subsubsection{Crosslinking and clustering of receptors}

The second and more general approach to increase internalisation and trafficking to the lysosomes consists in simultaneously targeting two different epitopes on the same antigen with a bispecific entity (e.g. a biparatopic antibody). This can not only increase selectivity towards cancer cells but it can also lead to enhanced internalisation and trafficking to the lysosome by inducing clustering and cross-linking of receptors [38, 39]. The past few years have seen an increase in the development of biparatopic platforms to promote receptor lysosomal trafficking; this approach is now also gathering momentum in the ADC field (Figure 2).

By combining two non-overlapping HER2-targeted antibodies, Li et al. recently developed a bivalent biparatopic antibody-like bispecific with two engineered cysteines in the Fc region to enable site-specific functionalisation with a potent tubulysin warhead [38]. The generated ADC was shown to promote HER2 clustering and lysosomal trafficking, and demonstrated potent target-mediated cytotoxic activity. Importantly, when compared to monospecific ADC T-DM1, not only the biparatopic ADC was more potent in tumour models representing T-DM1-eligible patients with high HER2 expression, but it also demonstrated potent, target-mediated cytotoxic activity in a panel of cancer cell lines expressing different levels of HER2 including in tumour model with low expression of HER2. The biparatopic ADC was 


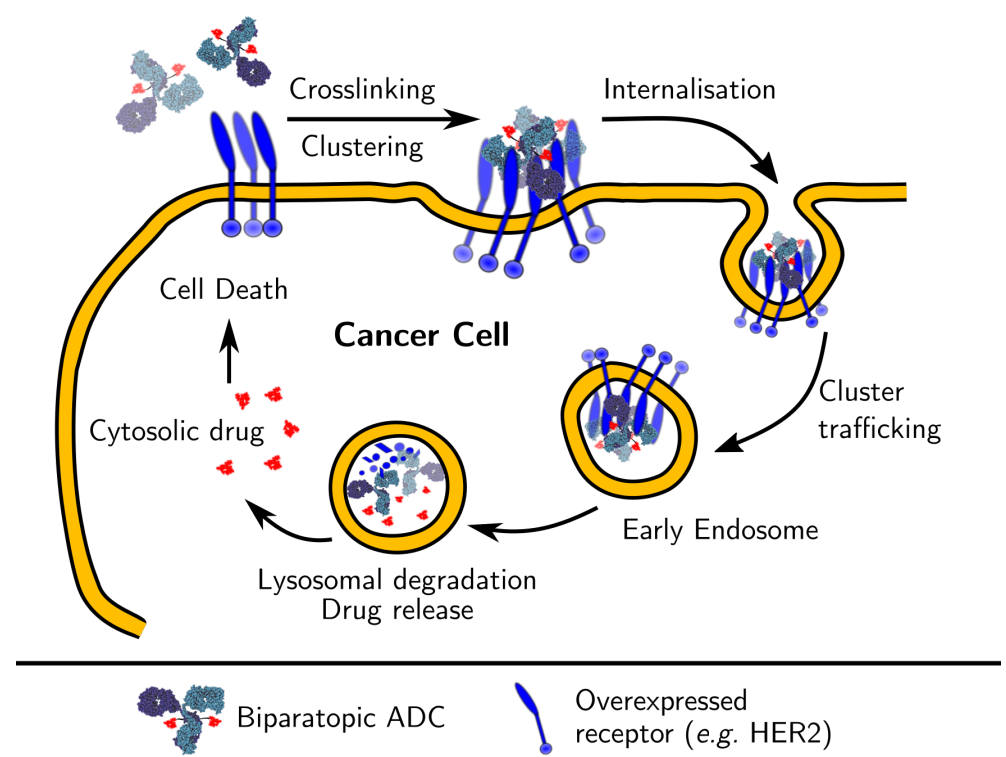

Figure 2: Schematic mechanism of action of biparatopic ADCs relying on crosslinking and clustering of receptors to enhance endocytosis and trafficking to the lysosome to deliver their payload.

also active in tumour models with acquired resistance to T-DM1.

Similarly, Hamblett et al. recently presented ZW49, a novel anti-HER2 biparatopic ADC that targets the same domains as trastuzumab (ECD4) and pertuzumab (ECD2) [39]. ZW49 was generated by conjugating the interchain reduced disulfide bonds of the parent naked biparatopic antibody to a novel $N$-acyl sulphonamide auristatin payload via a protease cleavable linker. The authors reported that the biparatopic ADC ZW49 internalised and trafficked to lysosomes in HER2-expressing cells to greater levels and faster than monospecific ADC (i.e. T-DM1). Superior tumour growth inhibition relative to other HER2 targeted therapies in low HER2 patient-derived xenograft model and in a trastuzumab-resistant high HER2 model was also demonstrated. Following these preclinical results, ZW49 was selected to move forward into a phase I study. 


\section{Conclusion}

After a difficult start in the 1980s, bispecific antibodies are now gathering great momentum. With required dose per patient several orders of magnitude lower than for monoclonal antibodies, clinical data obtained in oncology with BsAbs are impressive [22]. The ability to target two molecules using a single entity as well as the multiplicity of formats of such entities enable a variety of novel therapeutic approaches. With recent progress in protein engineering, fine tuning of bispecifics is now possible. It allows for a certain degree of tailoring in the binding partners to expand the therapeutic window of next-generation ADCs. In addition to better selectivity, internalisation and trafficking to the desired intracellular compartments, many bispecific ADCs have been shown to limit resistance and were still active in cell lines resistant to the parent monospecific ADC [32, 38-40]. As bispecifics ADCs are now entering the clinic, the improvement in activity and safety is yet to be fully determined as some of their constituent could still profit from recent advances in ADC technologies (e.g. site-specific conjugation to limit heterogeneity) [34]. Nevertheless, it is highly likely that an overall clinical benefit will be demonstrated in the close future. Research efforts in ADC and bispecific are merging; their synergy is generating novel strategies with high potential to get us one step up in the quest of the "magic bullet".

\section{References}

[1] Ducry, L.. Antibody-Drug Conjugates. volume 1045 of Methods in Molecular Biology. Springer, 2013. doi:10.1007/978-1-62703-541-5.

[2] Kohrt, H.E., et al. Anti-kir antibody enhancement of anti-lymphoma activity of natural killer cells as monotherapy and in combination with anti-cd20 antibodies. Blood 2014;123(5):678-86. URL: http://www.ncbi.nlm.nih.gov/pubmed/24326534. doi:10.1182/blood-2013-08-519199.

[3] Henricks, L.M., Schellens, J.H., Huitema, A.D., Beijnen, J.H.. The use of combinations of monoclonal antibodies in clinical oncology. Cancer Treat Rev 2015;41(10):859-867.

[4] Houot, R., Kohrt, H., Levy, R.. Boosting antibody-dependant cellular cytotoxicity against tumor cells with a cd137 stimulatory antibody. Oncoimmunology 2012;1(6):957-958. URL: http://www.ncbi. nlm.nih.gov/pubmed/23162770. doi:10.4161/onci.19974. 
[5] Beck, A., Haeuw, J.F., Wurch, T., Goetsch, L., Bailly, C., Corvaia, N.. The next generation of antibody-drug conjugates comes of age. Discov Med 2010;10(53):329-39. URL: http://www.ncbi.nlm. nih.gov/pubmed/21034674.

[6] Staerz, U.D., Kanagawa, O., Bevan, M.J.. Hybrid antibodies can target sites for attack by t cells. Nature 1985;314(6012):628-31. URL: http://www.ncbi.nlm.nih.gov/pubmed/2859527.

[7] Milstein, C., Cuello, A.C.. Hybrid hybridomas and their use in immunohistochemistry. Nature 1983;305(5934):537-40. URL: http://www.ncbi.nlm.nih.gov/pubmed/6137772.

[8] Curnow, R.T.. Clinical experience with cd64-directed immunotherapy. an overview. Cancer Immunol Immunother 1997;45(3-4):210-5. URL: http://www.ncbi.nlm.nih.gov/pubmed/9435876.

[9] Peipp, M., Valerius, T.. Bispecific antibodies targeting cancer cells. Biochem Soc Trans 2002;30(4):50711. URL: http://www.ncbi.nlm.nih.gov/pubmed/12196124. doi:10.1042/.

[10] Canevari, S., et al. Bispecific antibody targeted t cell therapy of ovarian cancer: clinical results and future directions. J Hematother 1995;4(5):423-7. URL: http://www.ncbi.nlm.nih.gov/pubmed/8581379. doi:10.1089/scd.1.1995.4.423.

[11] Lamers, C.H., Bolhuis, R.L., Warnaar, S.O., Stoter, G., Gratama, J.W.. Local but no systemic immunomodulation by intraperitoneal treatment of advanced ovarian cancer with autologous t lymphocytes re-targeted by a bi-specific monoclonal antibody. Int J Cancer 1997;73(2):211-9. URL: http://www.ncbi.nlm.nih.gov/pubmed/9335445.

[12] Segal, D.M., Weiner, G.J., Weiner, L.M.. Bispecific antibodies in cancer therapy. Curr Opin Immunol 1999;11(5):558-62. URL: http://www.ncbi.nlm.nih.gov/pubmed/10508714.

[13] Weiner, L.M., et al. Binding and cytotoxicity characteristics of the bispecific murine monoclonal antibody 2b1. J Immunol 1993;151(5):2877-86. URL: http://www.ncbi.nlm.nih.gov/pubmed/8103070.

[14] Weiner, L.M., et al. Phase i trial of 2b1, a bispecific monoclonal antibody targeting c-erbb-2 and fc gamma riii. Cancer Res 1995;55(20):4586-93. URL: http://www.ncbi.nlm.nih.gov/pubmed/7553634.

[15] Link, B.K., Kostelny, S.A., Cole, M.S., Fusselman, W.P., Tso, J.Y., Weiner, G.J.. Anti-cd3based bispecific antibody designed for therapy of human b-cell malignancy can induce t-cell activation by antigen-dependent and antigen-independent mechanisms. Int J Cancer 1998;77(2):251-6. URL: http://www.ncbi.nlm.nih.gov/pubmed/9650561.

[16] Hartmann, F., et al. Treatment of refractory hodgkin's disease with an anti-cd16/cd30 bispecific antibody. Blood 1997;89(6):2042-7. URL: http://www.ncbi.nlm.nih.gov/pubmed/9058726.

[17] Valone, F.H., et al. Phase ia/ib trial of bispecific antibody mdx-210 in patients with advanced breast or ovarian cancer that overexpresses the proto-oncogene her-2/neu. J Clin Oncol 1995;13(9):2281-92. URL: http://www.ncbi.nlm.nih.gov/pubmed/7545221. doi:10.1200/JC0.1995.13.9.2281.

[18] Posey, J.A., et al. A pilot trial of gm-csf and mdx-h210 in patients with erbb-2-positive advanced 
malignancies. J Immunother 1999;22(4):371-9. URL: http://www.ncbi.nlm.nih.gov/pubmed/10404439.

[19] James, N.D., Atherton, P.J., Jones, J., Howie, A.J., Tchekmedyian, S., Curnow, R.T.. A phase ii study of the bispecific antibody mdx-h210 (anti-her2 x cd64) with gm-csf in her2+ advanced prostate cancer. Br J Cancer 2001;85(2):152-6. URL: http://www.ncbi.nlm.nih.gov/pubmed/11461069. doi:10.1054/bjoc. 2001.1878.

[20] Borchmann, P., et al. Phase 1 trial of the novel bispecific molecule h22xki-4 in patients with refractory hodgkin lymphoma. Blood 2002;100(9):3101-7. URL: http://www.ncbi.nlm.nih.gov/pubmed/12384405. doi:10.1182/blood-2001-12-0295.

[21] Brinkmann, U., Kontermann, R.E.. The making of bispecific antibodies. MAbs 2017;9(2):182-212. URL: http://www.ncbi.nlm.nih.gov/pubmed/28071970. doi:10.1080/19420862.2016.1268307.

[22] Bargou, R., et al. Tumor regression in cancer patients by very low doses of a t cell-engaging antibody. Science 2008;321(5891):974-7. URL: http://www.ncbi.nlm.nih.gov/pubmed/18703743. doi:10.1126/ science. 1158545.

[23] Chudasama, V., Maruani, A., Caddick, S.. Recent advances in the construction of antibody-drug conjugates. Nat Chem 2016;8(2):114-9. URL: http://www.ncbi.nlm.nih.gov/pubmed/26791893. doi:10.1038/nchem. 2415 .

[24] Damelin, M.. Innovations for next-generation antibody-drug conjugates. Cancer Drug Discovery and Development. New York, NY: Springer Science+Business Media, 2018.

[25] Beck, A., Goetsch, L., Dumontet, C., Corvaia, N.. Strategies and challenges for the next generation of antibody-drug conjugates. Nat Rev Drug Discov 2017;16(5):315-337. URL: http://www.ncbi.nlm. nih.gov/pubmed/28303026. doi:10.1038/nrd.2016.268.

[26] Hinrichs, M.J., Dixit, R.. Antibody drug conjugates: Nonclinical safety considerations. AAPS J 2015;17(5):1055-64. URL: http://www.ncbi.nlm.nih.gov/pubmed/26024656. doi:10.1208/ s12248-015-9790-0.

[27] Sellmann, C., et al. Balancing selectivity and efficacy of bispecific epidermal growth factor receptor (egfr) x c-met antibodies and antibody-drug conjugates. J Biol Chem 2016;291(48):25106-25119. URL: http://www.ncbi.nlm.nih.gov/pubmed/27694443. doi:10.1074/jbc.M116. 753491.

[28] Mazor, Y., et al. Enhanced tumor-targeting selectivity by modulating bispecific antibody binding affinity and format valence. Sci Rep 2017;7:40098. URL: http://www.ncbi.nlm.nih.gov/pubmed/28067257. doi:10.1038/srep40098.

[29] Robert, B., et al. Tumor targeting with newly designed biparatopic antibodies directed against two different epitopes of the carcinoembryonic antigen (cea). Int J Cancer 1999;81(2):285-91. URL: http://www.ncbi.nlm.nih.gov/pubmed/10188732.

[30] Austin, C.D., et al. Endocytosis and sorting of erbb2 and the site of action of cancer therapeutics 
trastuzumab and geldanamycin. Mol Biol Cell 2004;15(12):5268-82. URL: http://www.ncbi.nlm.nih. gov/pubmed/15385631. doi:10.1091/mbc. e04-07-0591.

[31] Friedman, L.M., et al. Synergistic down-regulation of receptor tyrosine kinases by combinations of mabs: implications for cancer immunotherapy. Proc Natl Acad Sci U S A 2005;102(6):1915-20. URL: http://www.ncbi.nlm.nih.gov/pubmed/15684082. doi:10.1073/pnas. 0409610102.

[32] Lee, J.M., et al. Novel strategy for a bispecific antibody: induction of dual target internalization and degradation. Oncogene 2016;35(34):4437-46. URL: http://www.nature.com/articles/onc2015514. doi:10.1038/onc.2015.514.

[33] Andreev, J., et al. Bispecific antibodies and antibody-drug conjugates (adcs) bridging her2 and prolactin receptor improve efficacy of her2 adcs. Mol Cancer Ther 2017;16(4):681-693. URL: http: //www.ncbi.nlm.nih.gov/pubmed/28108597. doi:10.1158/1535-7163.MCT-16-0658.

[34] de Goeij, B.E., et al. Efficient payload delivery by a bispecific antibody-drug conjugate targeting her2 and cd63. Mol Cancer Ther 2016;15(11):2688-2697. URL: http://www.ncbi.nlm.nih.gov/pubmed/ 27559142. doi:10.1158/1535-7163.MCT-16-0364.

[35] Pols, M.S., Klumperman, J.. Trafficking and function of the tetraspanin cd63. Exp Cell Res 2009;315(9):1584-92. URL: http://www.ncbi.nlm.nih.gov/pubmed/18930046. doi:10.1016/j.yexcr . 2008.09 .020 .

[36] DeVay, R.M., et al. Improved lysosomal trafficking can modulate the potency of antibody drug conjugates. Bioconjug Chem 2017;28(4):1102-1114. URL: http://www.ncbi.nlm.nih.gov/pubmed/ 28151644. doi:10.1021/acs . bioconjchem.7b00013.

[37] DeVay, R.M., Shelton, D.L., Liang, H.. Characterization of proprotein convertase subtilisin/kexin type 9 (pcsk9) trafficking reveals a novel lysosomal targeting mechanism via amyloid precursor-like protein 2 (aplp2). J Biol Chem 2013;288(15):10805-18. URL: http://www.ncbi.nlm.nih.gov/pubmed/23430252. doi:10.1074/jbc.M113.453373.

[38] Li, J.Y., et al. A biparatopic her2-targeting antibody-drug conjugate induces tumor regression in primary models refractory to or ineligible for her2-targeted therapy. Cancer Cell 2016;29(1):117-29. URL: http://www.ncbi.nlm.nih.gov/pubmed/26766593. doi:10.1016/j.ccell.2015.12.008.

[39] Hamblett, K.J., et al. AACR 2018 Proceedings: Abstracts 3914. CTI Meeting Technology, 2018.

[40] Waldron, N.N., Barsky, S.H., Dougherty, P.R., Vallera, D.A.. A bispecific epcam/cd133-targeted toxin is effective against carcinoma. Target Oncol 2014;9(3):239-49. URL: http://www.ncbi.nlm.nih. gov/pubmed/23900680. doi:10.1007/s11523-013-0290-9. 